\title{
Travel Time Approximation in Anisotropic Layered Media
}

-V Singh, Geodata Processing and Interpretation Centre, ONGC, Dehradun-248195,U.P India

- Sanjay Kumar, Southern Region Business Centre, ONGC, Chennai-6000 008, Tamil Nadu, India

\begin{abstract}
:
It is well known that the earth's subsurface is a layered sequence of different constituent media at a variety of scales. This layered sequence will be transversely isotropic with the axis of symmetry lying perpendicular to the layering. The elastic parameters of such equivalent medium of stack of thin layers can be derived analytically from the elastic properties of the constituent layers. The anisotropic parameters of a transversely isotropic equivalent medium which is composed of two isotropic materials have been derived and computed results are studied. It is observed that these parameters are highly anisotropic for the equivalent medium if two isotropic and homogeneous layers are having different shear moduli and different V/V ratios. To study the response of investigating seismic waves in such a medium, an effort has been made to derive the analytical expressions of group velocity, move out velocity and travel time of quasi-P (P-P), quasi-S (SV-SV) and transverse (SH-SH) waves. These analytical expressions have been computed and results are studied in detail. It is found that the response of seismic wave parameters changes significantly for different composition of two isotropic materials with significantly different shear moduli and different ( Vs/V) ratio which is clearly seen in the results of moveout velocity and moveout travel time for all the three waves. Finally, the implication of this study in various steps of seismic exploration has been discussed in depth.
\end{abstract}

\section{Introduction:}

With the advent of digital recording and growing high speed computers a new era has started in the seismic prospecting with the improved data quality and geophysicists are looking for finer and finer details in reservoirs through seismic attribute's in addition to structural mapping. More and more high-resolution $\mathrm{P}$-wave data are being acquired for waves traveling very close to vertical. The acquired data are processed and interpreted to delineate the thin beds having small areal extents by assuming isotropic model of earth's subsurface. Therefore, it is assumed that these waves will not be affected by lateral variation of earth's subsurface i.e, by transverse isotropy. However, lateral variation will affect seismic wave propagation for steeply dipping structures and for large source-receiver spacings where incidence angle is large with the normal to the reflector and with the vertical. In these situations, ignorance of anisotropy in conventional processing and interpretation of seismic data has lead to serious errors that have been either unrecognized or attributed to data acquisition and various processing problems. The importance of anisotropic phenomena in wave propagation, processing and interpretation of seismic data is now widely recognized by the exploration community after advent of multicomponent recordings, 3-D seismic surveys, crosswell seismics and vertical seismic profiling ( Helbig, 1994).

Most of the existing work on reflection seismology in anisotropic media has been carried out for transversely isotropic media with vertical axis of symmetry and it has been reported in the literature byvarious workers from time to time. Thomsen(1986) has derived a set of parameters that can be used to describe the transverse isotropy of a single layer and approximated travel time curves of a wave in a single anisotropic layer only by initial slope of $\mathrm{T}^{2}$ and $\mathrm{X}^{2}$ plots. Rommel (1993) has derived analytical expressions of offset dependent polynomials of moveout velocity and travel time using Thomson's (1986) parameters in a weakly transversely isotropic media and emphasized the inclusion of anisotropy in interpretation of the seismic data. Tsvankin and Thomsen (1994) have 
presented an analytical and numerical analyses of short and long spread moveouts for single and multilayered transversely isotropic media by using three term Taylor series expansion. Singh and Kumar (1995) have derived more accurate analytical expressions of ray velocity, move out velocity and travel time including the contribution of first order derivative of phase velocity with respect to phase angle for reflected quasi-P (P-P), quasi-S( SV-SV) and transverse (SH-SH) waves in a single transversely isotropic layer which was ignored by earlier workers. Numerical results of this study have shown that moveout velocity changes significantly at zero offset and at smaller as well as larger offset distances due to contribution of first order derivative of phase velocity which inturn results into the significant change in the travel time at finite offsets.

Thomson's(1986) anisotropic parameters of the equivalent medium of a stack of thin layers have been derived using the elastic properties of the constituent layers by Brittan et al. ,(1995) analytically. These parameters enables greater understanding of the layered sequences indicating to what degree, particular combination of materials will appear anisotropic to seismic experiments. Very recently, Benyman et al., (1997) have analyzed the range of Thomsen anisotropic parameters for finely layered vertical transversely isotropic (VT1) media and their usefulness as indicators of rapid spatial changes in fluid content in the layers for which Gassmann's (1951) equation shows that the effect of fluids influence only Lame' constant (c) and not the shear modulus(m). The elastic constants of VTI media defined in terms of composite elastic properties of layered sequences may also be very useful for investigating seismic waves whose wavelength is shorter than the thickness of layered sequences. The interpretation of travel time curves over a layered medium would give more physical insight if analytical expressions for such travel time in terms of anisotropy parameters of earth's subsurface can be derived more accurately.

Keeping this in view, in this study an attempt has been made to study the effect of anisotropy parameters of a layered sequence on vari- ous physical characteristics such as phase velocity, group velocity, move-out velocity and travel time of quasi-P (P-P), quasi-S (SV-SV) and transverse ( $\mathrm{SH}-\mathrm{SH})$ waves. It is found that various composition of two materials with different elastic properties of a layered sequence changes seismic wave parameters significantly which may lead to significant errors in representation of earth's subsurface if the presence of anisotropy due to thin layering is not accounted properly.

\section{Theoretical Background of Elastic Anisotropy:}

The wave equation in any media can be described by the equation of motion as

$$
r\left(d^{2} u_{i} \not d t^{2}\right)=d s_{i j} / d x_{j}
$$

where $p$ designates the density of the medium, $t$ is time, $x_{j}$ represents the position vector of the considered point in the medium, $u_{i}$ represents the components of displacement at this point due to deformation and $\mathrm{s}_{\mathrm{ij}}$ is the components of the stress tensor. For a linear, elastic and anisotropic material, Hook's law states that each component of the stress $s_{i j}$ is linearly proportional to the strain $e_{\mathrm{kl}}$ and is expressed by

$$
s_{i j}={ }_{k=1}^{3}{ }_{k=1}^{3}{ }_{x} C_{i j k l} e_{k l} i, j=1,2,3
$$

where $\mathrm{C}_{\mathrm{ijkl}}$, is a tensor of rank fonr in three dimensions and obeys the laws of tensor transformation. This elastic stiffness tensor completely characterizes the elasticity of the medium and has a total of 81 components. Here $s_{i j}$ and $\mathrm{e}_{\mathrm{kl}}$ are the general tensor of rank two in three dimensions and each one has 9 components. Symmetry of stresses and strains reduce the number of independent elastic constants from 81 to 36 for a tensor of rank four. The stiffness tensor $\mathrm{C}_{\mathrm{ijkl}}$ can be represented more compactly by $6 \mathrm{x} 6$ matrix $\mathrm{C}_{\mathrm{ab}}$ following Voigt recipe. Further, for a unique strain energy potential the number of independent nonvanishingly distinct elastic constants reduce from 36 to 21 . This is the maximum number of elastic constants that any linear elastic medium can have without loss of generality. Restriction imposed by symmetry consid- 
erations reduce the number of constants much further. Isotropic linear elastic materials having maximum symmetry are completely characterized by two independent elastic constants where as materials having triclinic symmetry ( lowest symmetry ) require all 21 elastic constants. For isotropic media the matrix $\mathrm{C}_{\mathrm{ab}}$ assumes the simple form

$\mathrm{C}_{\mathrm{ab}}=\left|\begin{array}{cccccc}\mathrm{C}_{11} & \mathrm{C}_{12} & \mathrm{C}_{12} & 0 & 0 & 0 \\ \mathrm{C}_{12} & \mathrm{C}_{11} & \mathrm{C}_{12} & 0 & 0 & 0 \\ \mathrm{C}_{12} & \mathrm{C}_{12} & \mathrm{C}_{11} & 0 & 0 & 0 \\ 0 & 0 & 0 & \mathrm{C}_{44} & 0 & 0 \\ 0 & 0 & 0 & 0 & \mathrm{C}_{44} & 0 \\ 0 & 0 & 0 & 0 & 0 & \mathrm{C}_{44}\end{array}\right|$ (3)

where $\mathrm{C}_{12}=\left(\mathrm{C}_{11}-\mathrm{C}_{22}\right)$. Here independent and nonzero elements are $\mathrm{C}_{11}$ and $\mathrm{C}_{44}$ only and they are related with Lame' parameter(c), Shear modulus(m) and Bulk modulus (K) of the medium by

$$
\begin{aligned}
& \mathrm{C}_{11}=(\mathrm{c}+2 \mathrm{~m})=\mathrm{K}+(4 / 3) \mathrm{m} \\
& \mathrm{C} 12=\mathrm{c} \quad \text { and } \\
& \mathrm{C} 44=\mathrm{m}
\end{aligned}
$$

The physical properties of the earth's subsurface are often assumed to be isotropic i.e., independent of direction. This is convenient for theoretical point of view but very far away from the reality. Preferred orientation of mineral grains or shapes of isotropic minerals, parallel fractures or microcracks controlled by regional stress field and thin bedding of isotropic layers in the earth's subsurface makes most physical parameters in the vertical direction different from those inhorizontal directions. This phenomena is known as anisotropy and such material is known as Transversely isotropic with vertical axis of symmetry which has been discussed in detail by Singh et al.,(1997a, b). Transversely isotropic ( Hexagonal ) symmetry is most often observed in shales which constitute about 75 percent of the clastic fills of the sedimentary basins of the world. A transversely isotropic elastic material is completely specified by five independent constants among 12 nonzero components. In terms of $\mathrm{C}_{\mathrm{ab}}$ the elastic constants can be represented in matrix form as

$$
\mathrm{C}_{\mathrm{ab}}=\left|\begin{array}{cccccc}
\mathrm{C}_{11} & \mathrm{C}_{12} & \mathrm{C}_{13} & 0 & 0 & 0 \\
\mathrm{C}_{12} & \mathrm{C}_{11} & \mathrm{C}_{13} & 0 & 0 & 0 \\
\mathrm{C}_{13} & \mathrm{C}_{13} & \mathrm{C}_{33} & 0 & 0 & 0 \\
0 & 0 & 0 & \mathrm{C}_{44} & 0 & 0 \\
0 & 0 & 0 & 0 & \mathrm{C}_{44} & 0 \\
0 & 0 & 0 & 0 & 0 & \mathrm{C}_{66}
\end{array}\right|
$$

where $\mathrm{C}_{12}=\left(\mathrm{C}_{11}-2 \mathrm{C}_{66}\right)$, Here z-axis lies along the axis of symmetry.

\section{Anisotropic Parameters of a Layered Media:}

Propagation of seismic waves in anisotropic medium is different from the propagation in isotropic medium. When a seismic signal propagates in a stratified media, which is composed of elastically homogeneous and isotropic layers having small thickness as compared to the propagating seismic signal wavelength, then the layered medium can be replaced by an equivalent nondispersive transversely isotropic medium with axis of symmetry lying perpendicular to the layering. The elastic properties of this equivalent transversely isotropic medium can be derived algebraically from the elastic properties of the material that compose the layers (Backus, 1962). The conventional notations for transversely isotropic medium discussed in the previous section do not provide analytic insight about the hidden strength of anisotropy and condition for elliptically anisotropic medium on seismic wave signatures. An alternate notations for the five elastic constants of a transversely isotropic medium with vertical axis of symmetry were suggested by Thomsen(1986). The idea of Thomson's anisotropic parameters is to separate the influence of anisotropy from the isotropic quantities i.e., P- and S-wave velocities along the symmetry axis. Five elastic constants needed to describe the vertical transverse isotropy $\left(\mathrm{C}_{11}, \mathrm{C}_{33}, \mathrm{C}_{44}, \mathrm{C}_{66}\right.$ and $\mathrm{C}_{13}$ ) can be replaced by the vertical velocities $\mathrm{V}_{\mathrm{PO}}$ and $\mathrm{V}_{\mathrm{SO}}$ of $\mathrm{P}$ - and $\mathrm{S}$-waves respectively and three dimensionless parameters s, 5 and y. These new parameters can be written in terms of elastic modulus $\mathrm{C}_{\mathrm{ab}}$ as 


$$
\begin{gathered}
\left.\mathrm{V}_{\mathrm{PO}}{ }^{2}=\mathrm{C}_{33} / \mathrm{r}\right) \\
\mathrm{V}_{\mathrm{SO}}^{2}=\left(\mathrm{C}_{44} / \mathrm{r}\right) \\
\mathrm{e}^{=}\left[\mathrm{C}_{11}-\mathrm{C}_{13}\right] / 2 \mathrm{C}_{33}, \\
\mathrm{~d}=\left[\left(\mathrm{C}_{13}+\mathrm{C}_{44}\right)^{2}-\left(\mathrm{C}_{33}-\mathrm{C}_{44}\right)^{2}\right] \\
/\left[2 \mathrm{C}_{33}\left(\mathrm{C}_{33}-\mathrm{C}_{44}\right)\right] \\
\mathrm{C}_{11}, \mathrm{C}_{33}, \mathrm{C}_{44}, \mathrm{C}_{66} \text { and } \mathrm{C}_{13} \\
\mathrm{~g}=\left[\mathrm{C}_{66}-\mathrm{C}_{44}\right] /\left[2 \mathrm{C}_{44}\right]
\end{gathered}
$$

where $r$ is the density of the anisotropic medium.

Some of the advantages of Thomson's anisotropy parameters are as follows:

1.The dimensionless parameters ed, and g go to zero for isotropic media and therefore conveniently characterize the strength of anisotropy.

2. The parameter eclose to the fractional difference between horizontal and vertical P-wave velocities clearly defines the $\mathrm{P}$-wave anisotropy.

3. The parameter gclose to the fractional difference between horizontal and vertical SH-wave velocities clearly defines the $\mathrm{SH}$-wave anisotropy.

4. The parameter d responsible for near vertical $\mathrm{P}$-wave velocity variations. This is the parameter which determines the influence of anisotropy on short spread reflection data.

5. Elliptical anisotropy is a special case of transverse isotropy which is much simpler to understand through Thomson's anisotropy parameters than the conventional elastic constants. An elliptically anisotropic medium is characterized by elliptical P-wavefronts emanating from a point source. It is defined by condition

$$
e=d
$$

It has been observed that P-wave signatures are highly sensitive to the deviation from elliptical anisotropy i.e., for $(e-d)^{1} 0$. This makes $(e d)$ one of the most important parameters for identifying the rapid spatial changes in fluid content in the porous layers for which the effect of fluid only influence the Lame' constant (1) and not the shear modulus (m).

Brittan et al.,(1995) have derived Thomson parameters $\mathrm{V}_{\mathrm{PO}}, \mathrm{V}_{\mathrm{SO}}$, e, $\mathrm{d}$ and gin terms of composite elastic properties of two constituent layers of homogeneous isotropic materials and are written as

$$
\begin{aligned}
& \mathrm{V}_{\mathrm{PO}}^{2}=\left[\left\{\mathrm{fr} \mathrm{r}_{1}+(1-\mathrm{f}) \mathrm{r}_{2}\right\}\right. \\
& \left.\left\{\mathrm{fS}_{1}{ }^{2} / \mathrm{r}_{1}-(1-\mathrm{f}) \mathrm{S}_{2}{ }^{2} / \mathrm{r}_{2}\right\}\right]
\end{aligned}
$$

$$
\begin{aligned}
& \mathrm{V}_{\mathrm{SO}}^{2}=\left[\mathrm{fr}_{1}+(1-\mathrm{f}) \mathrm{r}_{2}\right\} \\
& \left\{\mathrm{ft}_{1}^{2} / \mathrm{r}_{1}-(1-\mathrm{f}) \mathrm{T}_{2}^{\left.\left.2 / r_{2}\right\}\right]}\right. \\
& e=\frac{\left[\mathrm{f}(1-\mathrm{f})\left(\mathrm{m}_{1}-\mathrm{m}_{2}\right)\left\{\left(\mathrm{l}_{1}-\mathrm{m}_{1}\right)-\left(\mathrm{I}_{2}+\mathrm{m}_{2}\right)\right\}\right]}{\left[\left(\mathrm{I}_{1}+2 \mathrm{~m}_{1}\right)\left(\mathrm{I}_{2}+2 \mathrm{~m}_{2}\right)\right]}
\end{aligned}
$$

$$
d=\frac{\left[2 f(1-f)\left(m_{1}-m_{2}\right)\left(l_{1} m_{1}+l_{2} m_{2}\right)\right]}{\left[\left(l_{1}+2 m_{1}\right)\left(l_{2}+2 m_{2}\right)\left\{m_{2} f+m_{1}(1-f)\right\}\right]}
$$

$$
g=\left[f(1-f)\left(m_{1}-m_{2}\right)^{2}\right] /\left[2 m_{1} m_{2}\right]
$$

where $r_{1}$ and $r_{2}$ are the densities, $m_{1}$ and $m_{2}$ are the shear modulus. $l_{1}$ and $l_{2}$ are the Lame' parameters of the two layers respectively. The $\mathrm{S}_{\mathrm{n}}{ }^{2}$ and $\mathrm{T}_{\mathrm{n}}{ }^{2}$ are defined as

$$
\begin{aligned}
& S_{n}^{2}=r_{n} /\left(I_{n}+2 m_{n}\right) \\
& T_{n}^{2}=r_{n} / m_{n} \quad \text { for } n=1 \text { or } 2
\end{aligned}
$$

The function $\mathrm{S}_{\mathrm{n}}^{2}$ represent square of $\mathrm{P}$ wave slowness for each layer, similarly $\mathrm{T}_{\mathrm{n}}{ }^{2}$ represent square of $\mathrm{S}$-wave slowness for each layer and d) is the volume fraction of material 1 present in the layered stack.

\section{Phase velocity:}

The elastic parameters defined in eqns.( 7a to 7e) for an equivalent transversely isotropic 
medium which is composed of two isotropic and homogeneous layers, can be used to construct the tensor $\mathrm{C}_{\mathrm{ijkl}}$, defined in eqn. $(1 \mathrm{~b})$ so that, the constitutive relation is known for the anisotropic medium. This results in three independent solutions of wave equation, one quasi-longitudinal, one quasi-transverse and one transverse for each direction of propagation. All the three are polarized in mutually orthogonal directions. The directional dependence of phase velocities of quasi-P(P-P), quasi-S ( SV-SV) and transverse (SH-SH) waves in terms of five elastic parameters (defined in eqns. 7a to 7e) are written as

$\mathrm{Vp}^{2}(q)=\mathrm{V}_{\mathrm{po}}^{2}\left[1+\operatorname{Sin}^{2} q+\mathrm{D}^{*}(q)\right]$

$\operatorname{Vsv}^{2}(q)=V_{\text {so }}^{2}\left[1+\left(\notin G^{2}\right) \operatorname{Sin}^{2} q-D^{*}(q) / G^{2}\right]$

$\mathrm{V}_{\mathrm{SH}}{ }^{2}(\mathrm{q})=\mathrm{V}_{\text {So }}{ }^{2}\left[1+2 \mathrm{gSin}{ }^{2} \mathrm{q}\right]$

Where

$$
\begin{aligned}
D^{*}(q)= & \left\{\left(1-G^{2}\right) / 2\right\}\left[\left\{1+4 d^{*} \operatorname{Sin}^{2} q \cos ^{2} q\right){ }^{2}{ }^{2} 1 / 2\right. \\
& /\left(1-G^{2}\right)^{2}+4\left(1-G^{2}+e\right) e \sin q /\left(1-G^{2}\right\}^{1 / 2}
\end{aligned}
$$

$\mathrm{G}=\left(\mathrm{V}_{\mathrm{SO}} / \mathrm{V}_{\mathrm{PO}}\right)$, $\mathrm{q}$ is the phase angle between wavefront normal and vwertical axis of symmetry and $d^{*}=\left[(2 d-e)\left(1-G^{2}\right)\right]$

Under weak anisotropic approximation i.e., for $e \ll 1, d \ll 1$ and $g \ll 1$, The expressions of the phase velocities of quasi-P, quasi-S and transverse waves are written as

$$
\begin{aligned}
& \mathrm{V}_{\mathrm{P}}(\mathrm{q})=\mathrm{V}_{\mathrm{PO}}\left[1+2 \mathrm{dSin}^{2} \mathrm{q} \operatorname{Cos}^{2} \mathrm{q}+2(\mathrm{e}+\mathrm{a}) \operatorname{Sin}^{4} \mathrm{q}\right]^{1 / 2} \\
& \mathrm{~V}_{\mathrm{SV}}(\mathrm{q})=\mathrm{V}_{\mathrm{SO}}\left[1+2(\mathrm{e}-\mathrm{a}) \mathrm{G}^{2}\right\} \operatorname{Sin}^{2} \mathrm{q} \operatorname{Cos}^{2} \mathrm{q}- \\
& \left.\left\{2 \mathrm{a} / \mathrm{G}^{2}\right\} \operatorname{Sin}^{4} \mathrm{q}\right] \\
& \mathrm{V}_{\mathrm{SH}}(\mathrm{q})=\mathrm{V}_{\mathrm{SO}}\left[1+2 \mathrm{~g} \operatorname{Sin}^{2} \mathrm{q}\right]^{1 / 2}
\end{aligned}
$$

Where $a=e^{2} /\left\{2\left(1-G^{2}\right\}\right.$

\section{Group Velocity and Group Angle:}

In an anisotropic medium, the waves are

not necessarily perpendicular to the wavefronts. Since we are using ray path for computation of travel time, we need the velocity which provide the velocity of energy transport or the ray velocity at ray angle. It depends on the phase velocity at phase angle $q$. The group velocity for a wave in anisotropic medium is given in terms of phase velocity magnitude as

$\mathrm{V}_{\mathrm{GI}}^{2}(\mathrm{y})=\mathrm{V}_{\mathrm{I}}^{2}(\mathrm{q})\left[1+\left\{1 / \mathrm{V}_{1}(\mathrm{q})\right\}\left(\mathrm{dV}_{1}(\mathrm{q}) / \mathrm{d}(\mathrm{q})\right]^{2}(10)\right.$

Here $\mathrm{V}_{\mathrm{I}}(\mathrm{q})$ is the magnitude of phase velocity and $y$ is the ray propogation angle from the source point to the wave front which gives the direction of energy propagation. For isotropic medium, the ray velocity and ray angle are equal to the phase velocity and phase angle. Here subscript "I" represents quasi-P (P-P), quasi-S (SV-SV) and transverse (SH-SH) waves respectively. Substituting the values of phase velocities and first order derivative of phase velocity with phase angle after certain simplification, the generalized expression for group velocity can be written in rearranged form as

$$
\begin{aligned}
\mathrm{V}_{\mathrm{GI}}{ }^{2}(\mathrm{y}) & =\mathrm{V}_{\mathrm{IO}}{ }^{2}\left[1+\mathrm{C}_{11} \operatorname{Sin}^{2} \mathrm{q}+\mathrm{C}_{12} \operatorname{Sin}^{4} \mathrm{q}+\mathrm{C}_{13} \operatorname{Sin}^{6} \mathrm{q}\right. \\
& +\mathrm{C}_{14} \operatorname{Sin}^{8} \mathrm{q}+\mathrm{C}_{15} \operatorname{Sin}^{10} \mathrm{q}+\mathrm{C}_{16} \operatorname{Sin}^{12} \mathrm{q}
\end{aligned}
$$

where

$$
\begin{aligned}
& C_{11}=A_{11}+A_{11}^{2} \\
& C_{12}=A_{12}+4 A_{11} A_{12}-A_{11}^{2}-A_{11}^{3} \\
& C_{13}=4 A_{12}^{2}-4 A_{11} A_{12}-5 A_{11}^{2} A_{12}+A_{11}^{3} \\
& C_{14}=4 A_{12}^{2}-4 A_{11} A_{12}-5 A_{11}^{2} A_{12}+A_{11}^{3} \\
& C_{15}=8 A_{11}^{2} A_{12}-4 A_{12}^{3} \\
& C_{16}=4 A_{12}{ }^{3}
\end{aligned}
$$$$
\begin{aligned}
& A_{P 1}=2 d \\
& A_{P 2}=2(e-d+a)
\end{aligned}
$$

Here, for quasi-P (P-P) waves 
for quasi-S (SV-SV) waves

$$
\begin{aligned}
& A_{\mathrm{sv} 1}=2\left(e-d / G^{2}\right. \\
& A_{s v 2}=2(e-d+a) / G^{2}
\end{aligned}
$$

and for transverse (SH-SH) waves

$$
\mathrm{A}_{\mathrm{SH} 1}=2 \mathrm{~g}
$$

$$
\mathrm{A}_{\mathrm{SH} 2}=0
$$

The relationship between ray and phase angle in anisotropic medium is given by

$$
\begin{aligned}
& \tan y=\tan q\left[1+1 /(\sin q \cos q)\left\{\left(1 / V_{1}(q)\right)\right.\right. \\
& \left.\left(d V_{1}(q) / d(q)\right\}+\left(\left(1+V_{1}(q)\right)\left(d_{1}(q) / d q\right)\right\}^{2}\right]
\end{aligned}
$$

Using phase velocity expressions given in eqns (9) and first derivative of phase velocity with phase angle, we can obtain analytical expression of ray angle for the corresponding wave which can be written in generalized form as

$\tan (\mathrm{y})=$

$\tan q\left[1+\frac{\left(\mathrm{A}_{11}+2 \mathrm{~A}_{12} \operatorname{Sin}^{2} \mathrm{q}\right)}{\left(1+\mathrm{A}_{11} \operatorname{Sin}^{2} \mathrm{q}+\mathrm{A}_{12} \operatorname{Sin}^{4} \mathrm{q}\right)}+\frac{\operatorname{Sin}^{2}{ }^{2} \operatorname{Cos}^{2} \mathrm{q}\left(\mathrm{A}_{11}+2 \mathrm{~A}_{12} \operatorname{Sin}^{2} \mathrm{q}\right)^{2}}{\left(1+\mathrm{A}_{11} \operatorname{Sin}^{2} \mathrm{q}+\mathrm{A}_{12} \operatorname{Sin}^{4} \mathrm{q}\right)}\right.$

For $q=0^{\circ}$ and $q=90^{\circ}$ the derivative of phase velocity becomes zero and for these cases group velocity becomes equal to phase velocity and group angle equals to phase angle which is the case of isotropic media.

\section{Moveout Velocity:}

Singh and Kumar (1995) demonstrated the effect of angular dependence of velocity on travel time with a single homogeneous anisotropic and horizontal layer and shown that slope of travel time function varies with an angle of incidence or equivalently with offset distance between source and receiver. The general equation governing the slope afall offset distances is given as

$$
\frac{\mathrm{dt}^{2}}{\mathrm{dX}^{2}}=\frac{1}{\mathrm{~V}_{\text {IMO }}^{2}}=\frac{1}{\mathrm{~V}_{\text {GI }}^{2}}\left[1-\frac{2 \cos ^{2} \mathrm{y} \mathrm{dV}_{\mathrm{GI}}(\mathrm{y})}{\mathrm{V}_{\mathrm{GI}}(\mathrm{y}) \mathrm{dSin}^{2} \mathrm{y}}\right]
$$

where $\mathrm{v}_{\mathrm{GI}}(\mathrm{y})$ represents the group velocity and
$\mathrm{V}_{\text {IMO }}$ represents the local moveout velocity of the corresponding seismie wave. Brown et al., (1991) have shown that for weakly anisotropic medium, the difference between phase angle and ray angle is of second order and therefore the ray angle can be equated to phase angle. Replacing phase angle $q$ by ray angle in eqn. (11), the expression for the ray velocity can be obtained which is directly dependent on ray angle. After having direct dependence of ray velocity on ray angle the computation of moveout velocity at any offset is straight forward. The general expression for moveout velocity is written as

$$
\begin{aligned}
& \mathrm{V}_{\mathrm{IMO}}^{2}=\mathrm{V}_{\mathrm{IO}}^{2}\left[1+\mathrm{C}_{11}+2 \mathrm{C}_{12} \operatorname{Sin}^{2} \mathrm{y}\right. \\
& +\left(3 \mathrm{C}_{13}-\mathrm{C}_{12}\right) \operatorname{Sin}^{4} \mathrm{y}+\left(4 \mathrm{C}_{14}-2 \mathrm{C}_{13}\right) \operatorname{Sin}^{6} \mathrm{y} \\
& \left.+\left(5 \mathrm{C}_{15^{-}}-3 \mathrm{C}_{14}\right) \operatorname{Sin}^{8} \mathrm{y}+\left(6 \mathrm{C}_{6}-4 \mathrm{C}_{15}\right) \operatorname{Sin}^{10} \mathrm{y}\right) \\
& \left.+\left(-5 \mathrm{C}_{16}\right) \operatorname{Sin}^{12} \mathrm{y}\right]
\end{aligned}
$$

Since ray angles are of secondary interest, the moveout velocities can be written in terms of offset $(X)$ and desired reflector depth $(Z)$. For ray angle $y=\tan ^{-1}(X / 2 Z) £ 45^{\circ}$, $\operatorname{Sin}^{2} y$ can be written as $\operatorname{Sin}^{2} \mathrm{y}=\left[\mathrm{X}_{1}{ }_{1}^{2} /\left(1+\mathrm{X}_{1}{ }^{2}\right)\right] \sim\left[\mathrm{X}_{1}{ }^{2}-\mathrm{X}_{1}{ }^{4}+\mathrm{X}_{1}{ }^{6}-\mathrm{X}_{1}{ }^{8}+\mathrm{X}_{1}{ }^{10}-\mathrm{X}_{1}{ }^{12}+\ldots\right]$ (16) where $\mathrm{X}_{1}=(\mathrm{X} / 2 \mathrm{Z})$.Substituting the values of $\operatorname{Sin}^{2} \mathrm{y}$, and its higher powers in the moveout velocity eqn.(15), we can obtain the final expression of moveout velocity which is written as

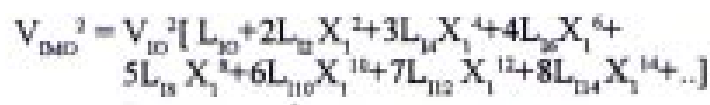

where

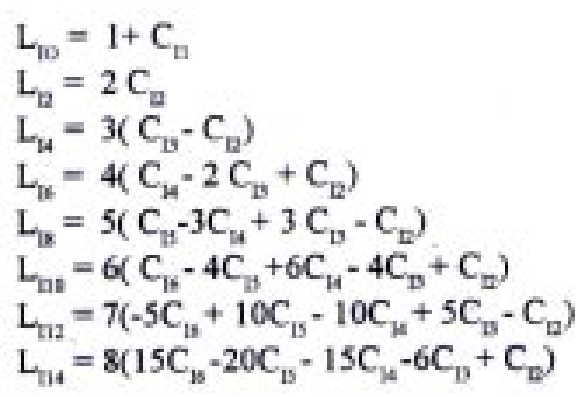

The above analytical expression of moveout velocity can be reduced to those given 
by Rommel (1993 ) by ignoring higher order contribution of anisotropic parameters. The general expression for normal moveout velocity can be obtained by retaining only first term of eqn.( 17) i.e., for the limit $X$---> 0 . we can obtain

$$
\mathrm{V}_{\mathrm{NMO}}^{2}=\mathrm{V}_{\mathrm{IO}}{ }^{2} \mathrm{~L}_{\mathrm{IO}}
$$

Substituting the value of coefficient $\mathrm{L}_{\mathrm{IO}}$, we can obtain the NMO velocity for the particular wave which reduces to those reported by Thomson (1986) if higher order terms of anisotropic parameters are neglected. After retaining the first term only the expressions for normal moveout velocity of quasi-P (P-P), quasi-S (SV$\mathrm{SV}$ ) and transverse(SH-SH) waves for weak anisotropy reduces to

$$
\begin{aligned}
& \mathrm{V}_{\mathrm{van}}(\mathrm{P})=\mathrm{V}_{0}(1+\delta) \quad 19(\mathrm{a}) \\
& \mathrm{V}_{\operatorname{san}}(\mathrm{SV})=\mathrm{V}_{\mathrm{so}}\left\{1+(\varepsilon-\delta) / \mathrm{G}^{2}\right\} \quad 19(\mathrm{~b}) \\
& \mathrm{V}_{\mathrm{san}}(\mathrm{SH})=\mathrm{V}_{\mathrm{so}}(1+\gamma) \quad 19(\mathrm{c})
\end{aligned}
$$

The last result of normal moveout velocity for transverse (SH-SH) waves does not require the limit $\mathrm{X}$-» 0 , i.e.. The $\mathrm{t}^{2}-\mathrm{X}^{2}$ graph gives straight line as it gives in case of isotropic media. This is the well known result for elliptical wavefronts (Van der Stoep, 1966).

\section{Travel Time Approximation:}

After having approximation of moveout velocity or reciprocal slope of travel time curve, the travel time itself can be computed by integration over the offset distance. In this integration, constant of integration is the square of vertical travel time $\left(\mathrm{t}_{\mathrm{IO}}\right)$

$$
\mathrm{t}_{1}^{2}=\mathrm{t}_{00}{ }^{2}+\int_{0}^{\mathrm{x}^{2}} \frac{1}{\mathrm{~V}_{\mathrm{MO}^{2}}{ }^{2}(\mathrm{X})} d \mathrm{X}^{2}
$$

Substituting the values of moveout velocity from eqn.(17) we can obtain the general expression of travel time which is written as

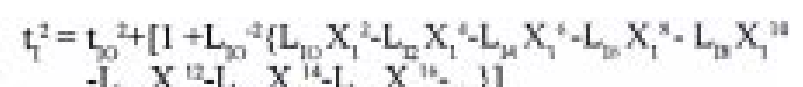

Here $t_{\mathrm{IO}}=\left(2 \mathrm{Z} / \mathrm{V}_{\mathrm{IO}}\right)$. Above generalized travel time equation reduces to those given by Rommel (1993) by ignoring higher order contri- bution of anisotropic parameters.

\section{Results and Discussion:}

Using analytical expressions of anisotropic parameters Vpo, Vso and three dimensionless parameters $g, 5$ and $Y$ given in eqns. (7a,b,c,d and e) for a transversely isotropic medium which is composed of two homogeneous and isotropic materials have been computed for $\mathrm{m}_{1}=8.36 \times 10^{9}$ $\mathrm{Nm}^{-2}, r_{1}=2500 \mathrm{~kg} \mathrm{~m}^{-3}, r_{1}=r_{2}, r_{1}=m_{1}$, and $l_{2}$ $=0.05 l_{1}$ for different values of volume fraction (f) of material 1 for different ratio of shear moduli and results are shown in Figs ( 1,2,3 and 4). P wave vertical decreases with increasing volume fraction ( $\mathrm{f}$ ) of material 1 for $\left(\mathrm{m}_{1} / \mathrm{m}_{2}\right)<<1$ where as as it increases exponentially for $\left(\mathrm{m}_{1} / \mathrm{m}_{2}\right)>>1$ as shown in Fig.(l a). The variation in S- wave vertical velocity follows similar trend as described for P-wave vertical velocity (Fig. 1 b).

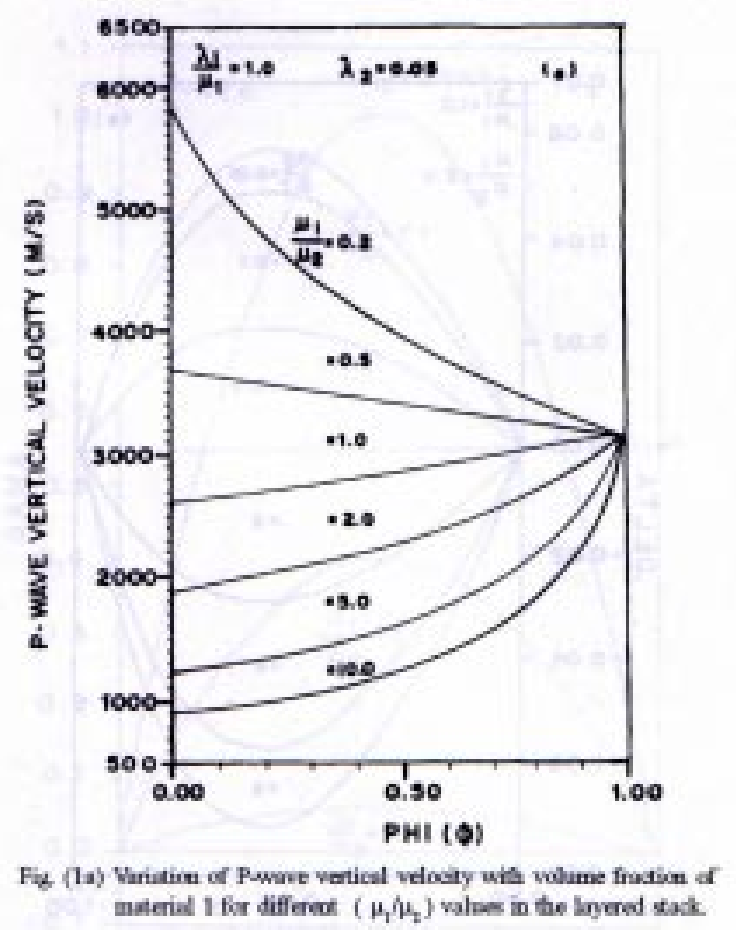

Fig 1 (a) Variation of $P$ Wave vertical velocity with volume fraction of material 1 for different $\left(\mathrm{m}_{1} / \mathrm{m}_{2}\right)$ values in the layered stack.

The variation of anisotropic parameter $\mathrm{S}$ with volume fraction ( $f$ ) for different values of shear moduli is shown in Fig.(2a) and for different values of $\left(l_{2} / m_{2}\right)$ at $\left(m_{1} / m_{2}\right)=2$ is shown in Fig.(2b). The values of anisotropy parameter $d$ increases with volume fraction ( $f$ ) and becomes maximum for a particular volume fraction. With 


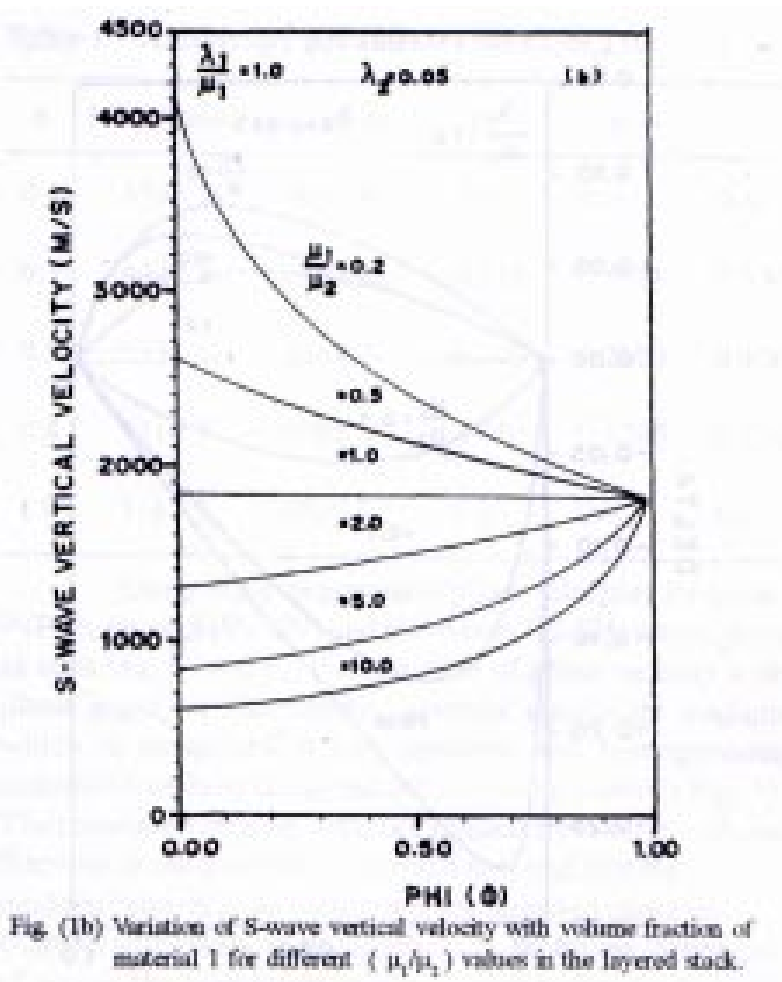

Fig 1 (b) Variation of S Wave vertical velocity with volume fraction of material 1 for different $\left(\mathrm{m}_{1} / \mathrm{m}_{2}\right)$ values in the layered stack.

the further increase of $f$, the value of anisotropy parameter $d$ decreases and becomes zero for $f=$ 1. Maximum value of d depend upon the ratio of shear moduli. For $\left(\mathrm{m}_{1} / \mathrm{m}_{2}\right)>1$, the anisotropy parameter $d$ is always positive and its maximum value shift towards higher value of volume fraction( $f)$ where as for $\left(m_{1} m_{2}\right)<1$, dis negative and maximum value shift towards lower $f$. The polarity of the anisotropy parameter $\mathrm{d}$ depends on $(\mathrm{l} / \mathrm{m})$ ratio of the two layers. It is found that the value of anisotropy parameter $\mathrm{d}$ becomes negative when the condition $\left(I_{1} / m_{1}\right)<\left(I_{2} / m_{2}\right)$ is satisfied. The value of $d$ becomes zero if either $\left(\mathrm{m}_{1} / \mathrm{m}_{2}\right)$ or $(\mathrm{Vs} / \mathrm{Vp})$ ratio of the two constituent layers becomes equal.

The variation of eand gwith volume fraction ( $\mathrm{f}$ ) are shown in Fig. (3a,3b and 4) respectively. The anisotropy parameters $e$ and $g$ becomes zero if shear moduli of two layers are equal. The actual magnitude of these parameters depends on the difference between shear moduli of two layers as it has been discussed in case of the anisotropy parameter $\mathrm{d}$. The maximum magnitude e and goccur at $f=0.5$ for all the values of $\left(m_{1} / m_{2}\right)$. This means that the factor $\{f(1-f)$ \}maximizes the value of eand gwhen there is an equal amount of material present. The value of anisotropy parameter e can be negative also if the difference between the sums of material 1's Lame' parameter and shear modulus $\left(l_{1}+m_{1}\right)$ becomes less than the sum of material 2 Lame' parameter and shear modulus $\left(l_{2}+m_{2}\right)$ i.e., $\left(l_{1}+\right.$ $\left.m_{1}\right)<\left(l_{2}+m_{2}\right)$ as it can be seen in Fig.(3b). This means that the layer must have largest bulk modulus and smallest shear modulus (i.e., the layer must be the most incompressible and least rigid). Fig. (4) clearly shows that the value ofy is always positive. This suggests that for a two layer sequence the velocity of transverse ( $\mathrm{SH}-\mathrm{SH}$ ) wave perpendicular to the axis of symmetry is always greater than or equal to the velocity of the S-wave parallel to the axis of symmetry. Thus, the computed results of five elastic parameters Vpo, Vso and three dimensionless parameters e, $\mathrm{d}$ and $\mathrm{g}$ for a transversely isotropic equivalent medium, composed of two homogeneous and isotropic layers have clearly demonstrated that to produce a highly anisotropic equivalent medium from a stack of two thin layers, the layers must have significantly different shear moduli and (Vs/Vp) ratio values.

Keeping this in view, an effort has been made to study the response of a seismic wave propagating through a transversely isotropic media which is composed of two isotropic and homogeneous materials, the numerical parameters used for this study are given in Table- 1 .

Using the expressions of phase velocities for quasi-P (P-P), quasi-S (SV-SV) and transverse(SH-SH) waves given in eqns. (9a, b and c), the variation of phase velocity with phase angle for transversely isotropic equivalent medium which is composed of two isotropic and homogeneous materials have been computed and results are shown in Fig.(5). The correlation of these velocities is made for different volume fractions of the material 1 (f) and it is found that for $0<f<1$, medium behaves as an anisotropic medium and phase velocity changes with phase angle significantly for all the three types of waves, the magnitude of phase velocity for $0<f<1$ lies between the phase velocity for $f=0$ and $f=1$. 


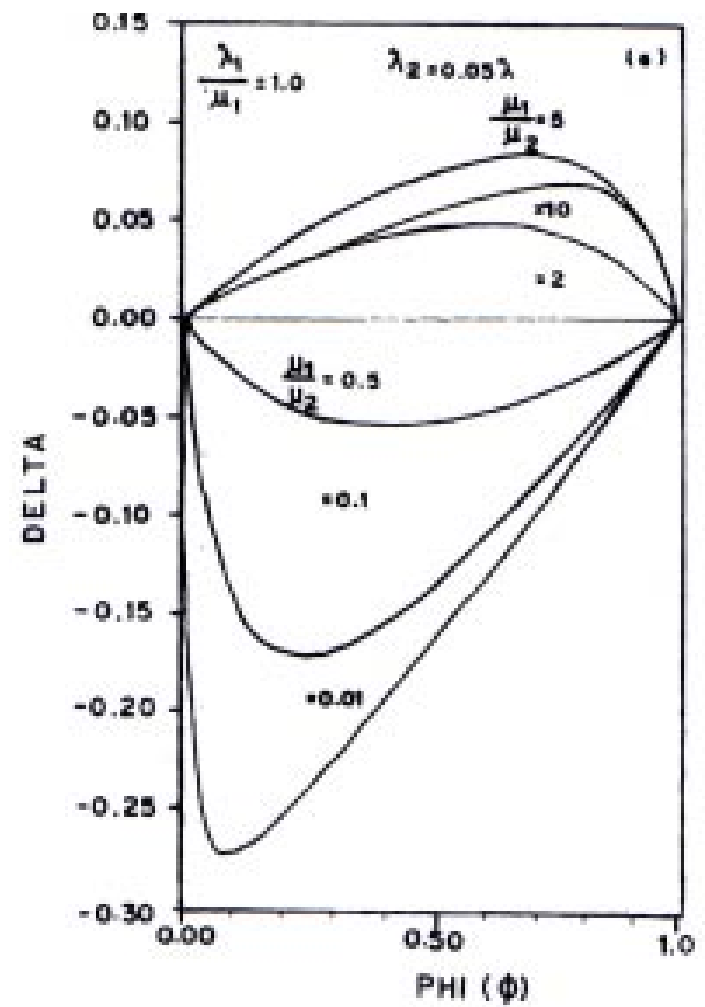

Fig 2 (a) Variation of Anisotropic Parameter d with volume fraction of material 1 for different $\left(\mathrm{m}_{1} / \mathrm{m}_{2}\right)$ values in the layered stack.

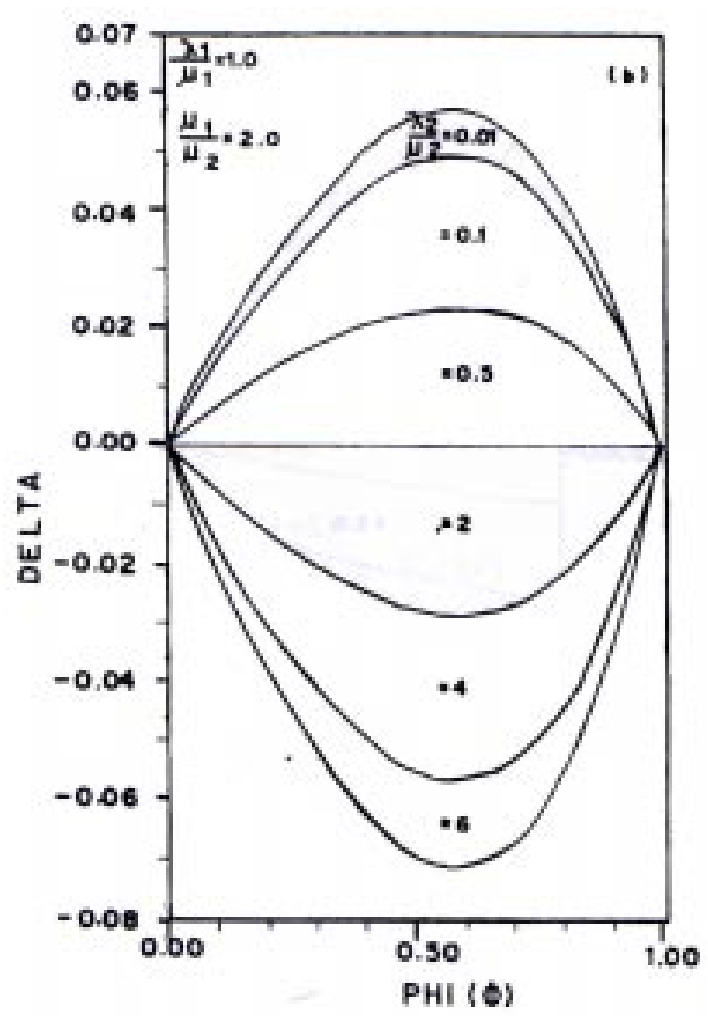

Fig 2 (b) Variation of Anisotropic Parameter $d$ with volume fraction of material 1 for different $\left(I_{2} / m_{2}\right)$ values in the layered stack.

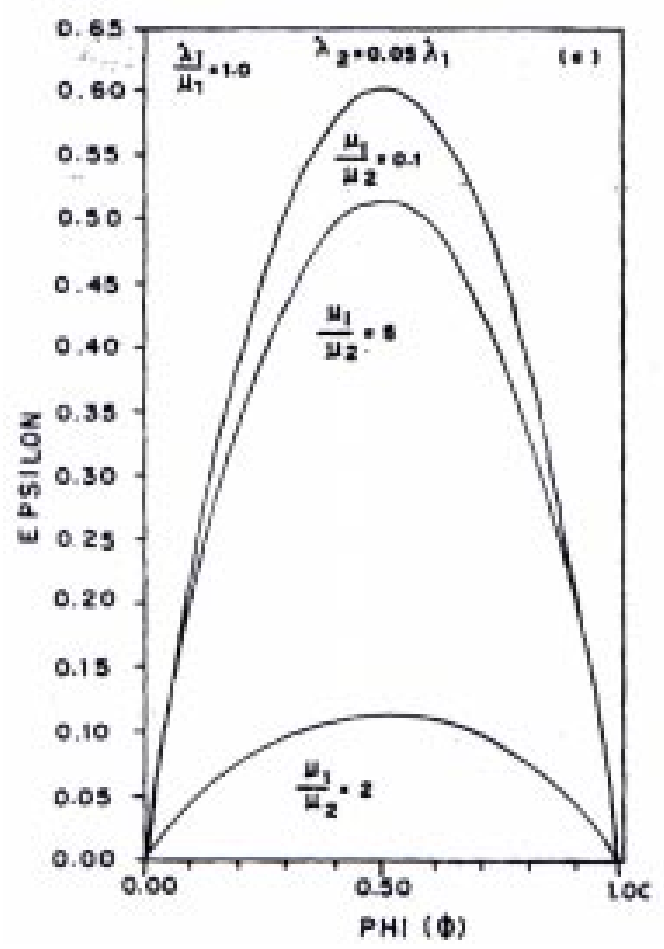

Fig 3 (a) Variation of Anisotropic Parameter e with volume fraction of material 1 for different $\left(\mathrm{m}_{1} / \mathrm{m}_{2}\right)$ values in the layered stack.

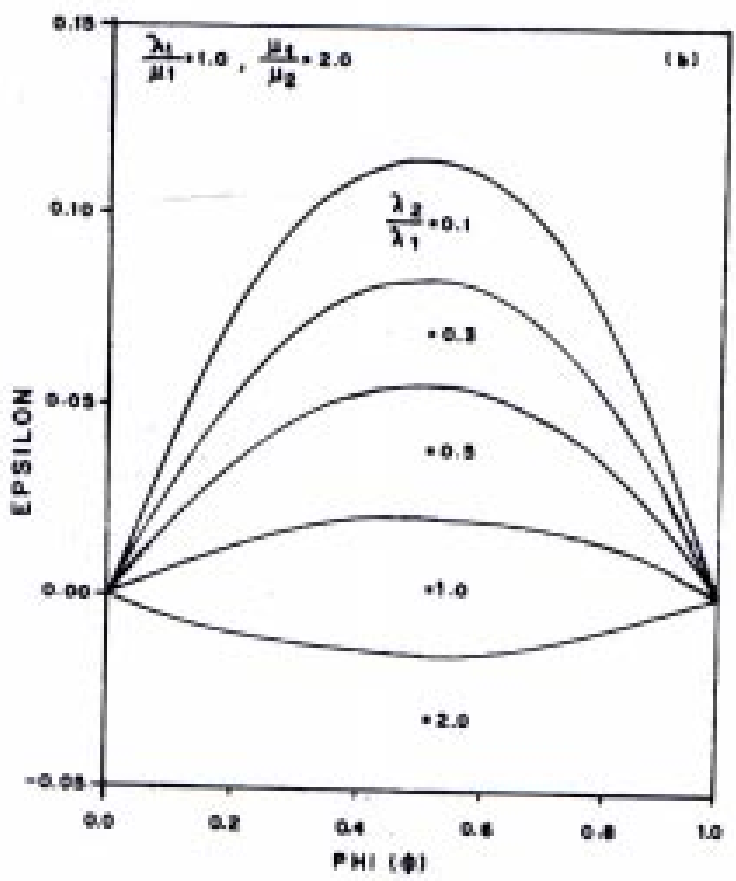

Fig 3 (b) Variation of Anisotropic Parameter e with volume fraction of material 1 for different $\left(l_{2} / l_{1}\right)$ values in the layered stack. 


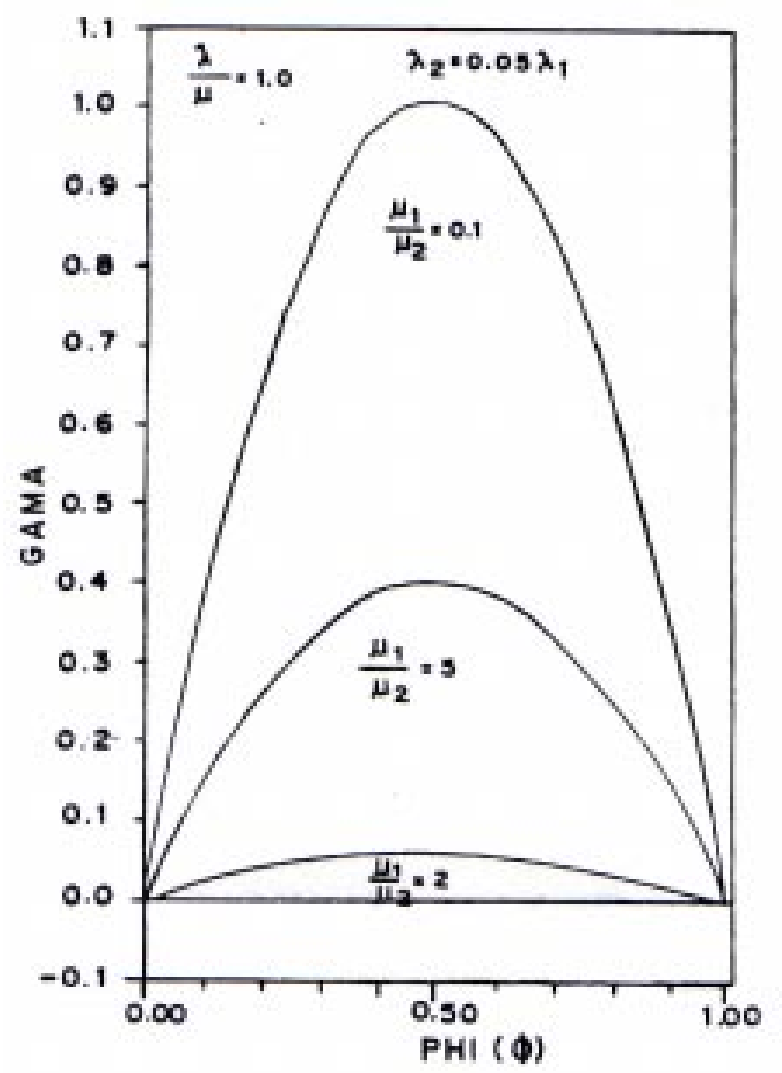

Fig 4 Variation of Anisotropic Parameter gwith volume fraction of material 1 for different $\left(\mathrm{m}_{1} / \mathrm{m}_{2}\right)$ values in the layered stack.
Table 1: Anisotropic parameter for $(\mathrm{m} 1 / \mathrm{m} 2)=2$

\begin{tabular}{llllll}
\hline$f$ & $V p o(m / s)$ & $V s o(m / s)$ & $d$ & $e$ & $g$ \\
\hline 0.0 & 5798.2 & 4089.8 & 0.0 & 0.0 & 0.0 \\
0.3 & 4440.2 & 2757.3 & -0.125 & 0.1700 & 0.336 \\
0.5 & 3931.6 & 2361.2 & -0.109 & 0.2023 & 0.400 \\
0.8 & 3416.7 & 1995.6 & -0.050 & 0.1295 & 0.256 \\
1.0 & 3167.9 & 1829.0 & 0.0 & 0.0 & 0.0 \\
\hline
\end{tabular}

The difference between phase angle and ray angle has been computed using the eqn. (13) for all the three waves namely quasi-P, quasiSand transverse waves. This difference has been plotted against phase angle for different volume fraction of material 1 (f) and results are shown in Fig.(6). For $f=0$ and $f=1$ the ray angle and phase angle becomes equal. As $f$ increases this difference increases from $-0.6^{\circ}$ to $2.7^{\circ}$ for $q<30^{\circ}$ and it increases from $7.2^{\circ}$ to $11^{\circ}$ for $q>30^{\circ}$ for quasi- $\mathrm{P}$ waves for a particular value of phase angle. With further increase in phase angle this difference reduces and becomes zero for $q=90^{\circ}$. In case of quasi-S waves this difference reduces
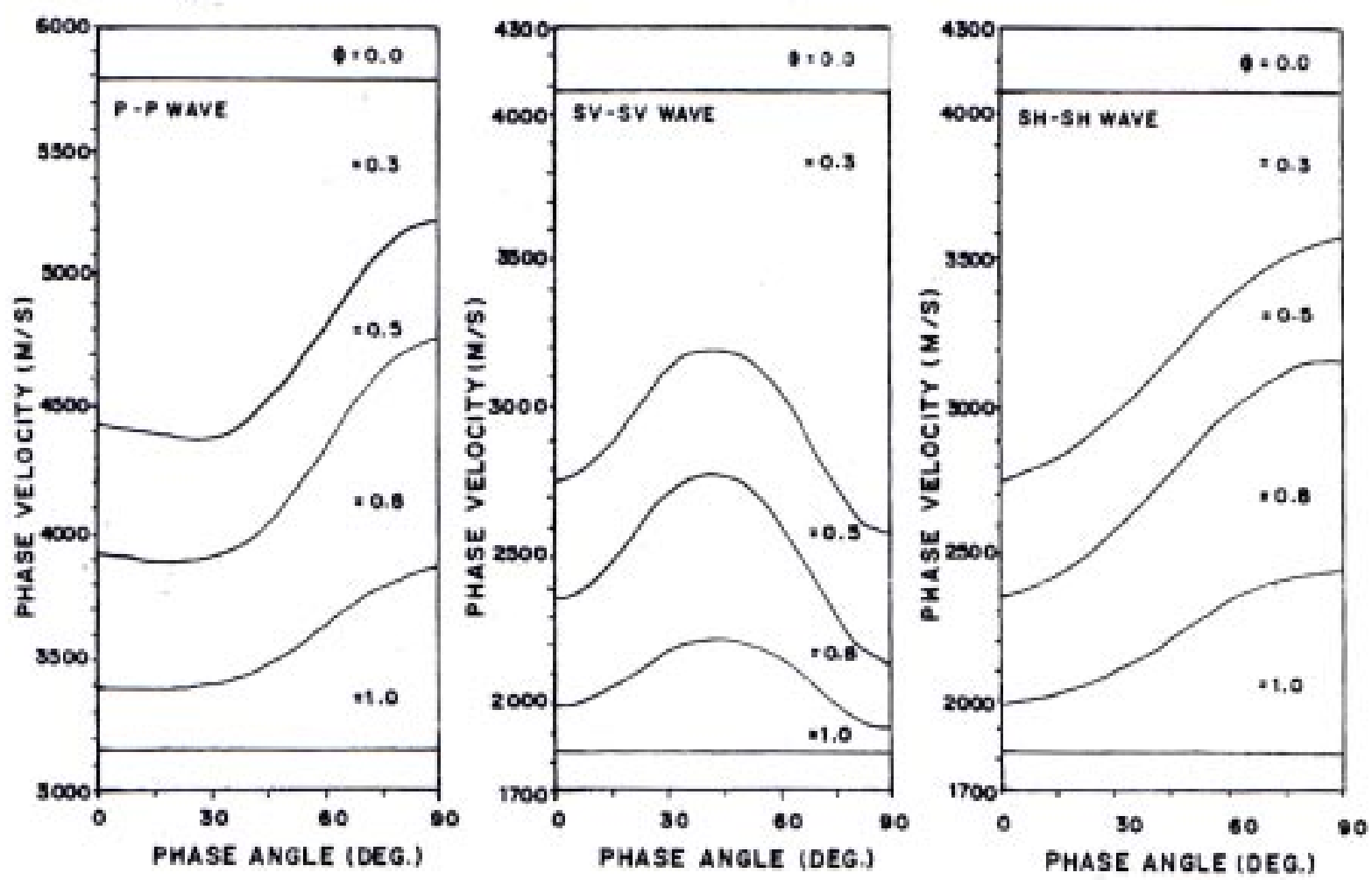

Fig 5 Variation of phase velocities with phase angle for different volume fraction of material 1 in the layered stack. 

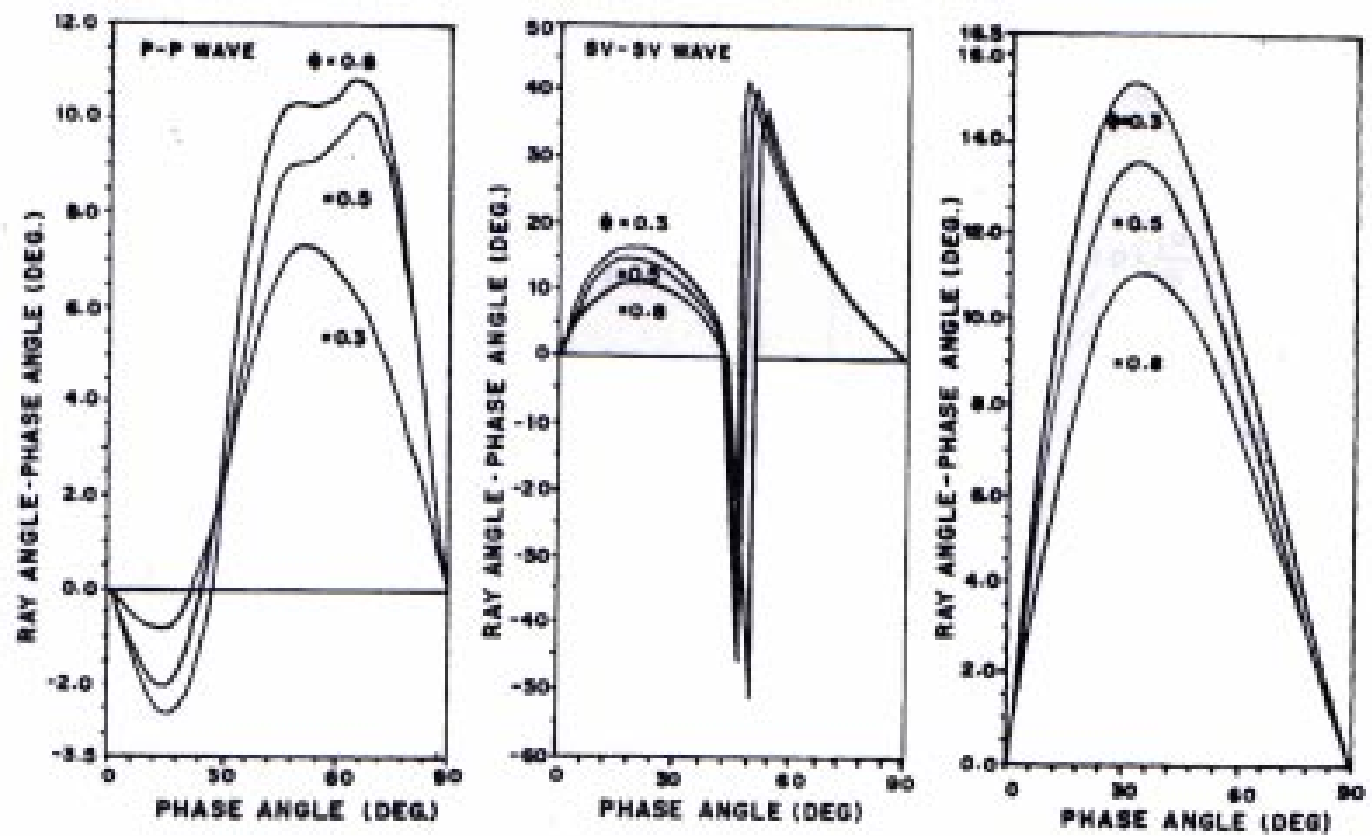

Fig 6. Variation of ray angle with phase angle for different volume fractions of material 1 in the layeredstack

from $17^{\circ}$ to $10^{\circ}$ as $f$ increases from 0.3 to 0.8 for $\mathrm{q}<30^{\circ}$ and it becomes zero at $\mathrm{q}=45^{\circ}$ for all the values of volume fraction of material 1ying in the range $0<f<1$. This difference oscillate between $-52^{\circ}$ to $43^{\circ}$ for the phase angle lying in the range $45<\mathrm{q}<54^{\circ}$ and with further increase of phase angle $q$ it reduces and become zero at $q$ $=90^{\circ}$. In case of transverse waves the difference between ray angle and phase angle is always positive for $0<\mathrm{f}<1$. As volume fraction of material 1 (f) increases from 0.3 to 0.8 the difference reduces from $15.5^{\circ}$ to $11.0^{\circ}$ at particular value of phase angle q. With the further increase in qit follows similar trend as in case of other two waves. It is seen that the difference of ray angle and phase angle is quite significant as $\mathrm{f}$ for higher values of phase angle (i.e., for $q>45^{\circ}$ ) and it is minimum to moderate for $q<45^{\circ}$

From the analytical expressions of group velocity and group angle given in eqns (11 and 13) it is seen that the difference of group angle and phase angle propagate into the second order for further analysis of moveout velocity and travel time approximation. For short spread and intermediate
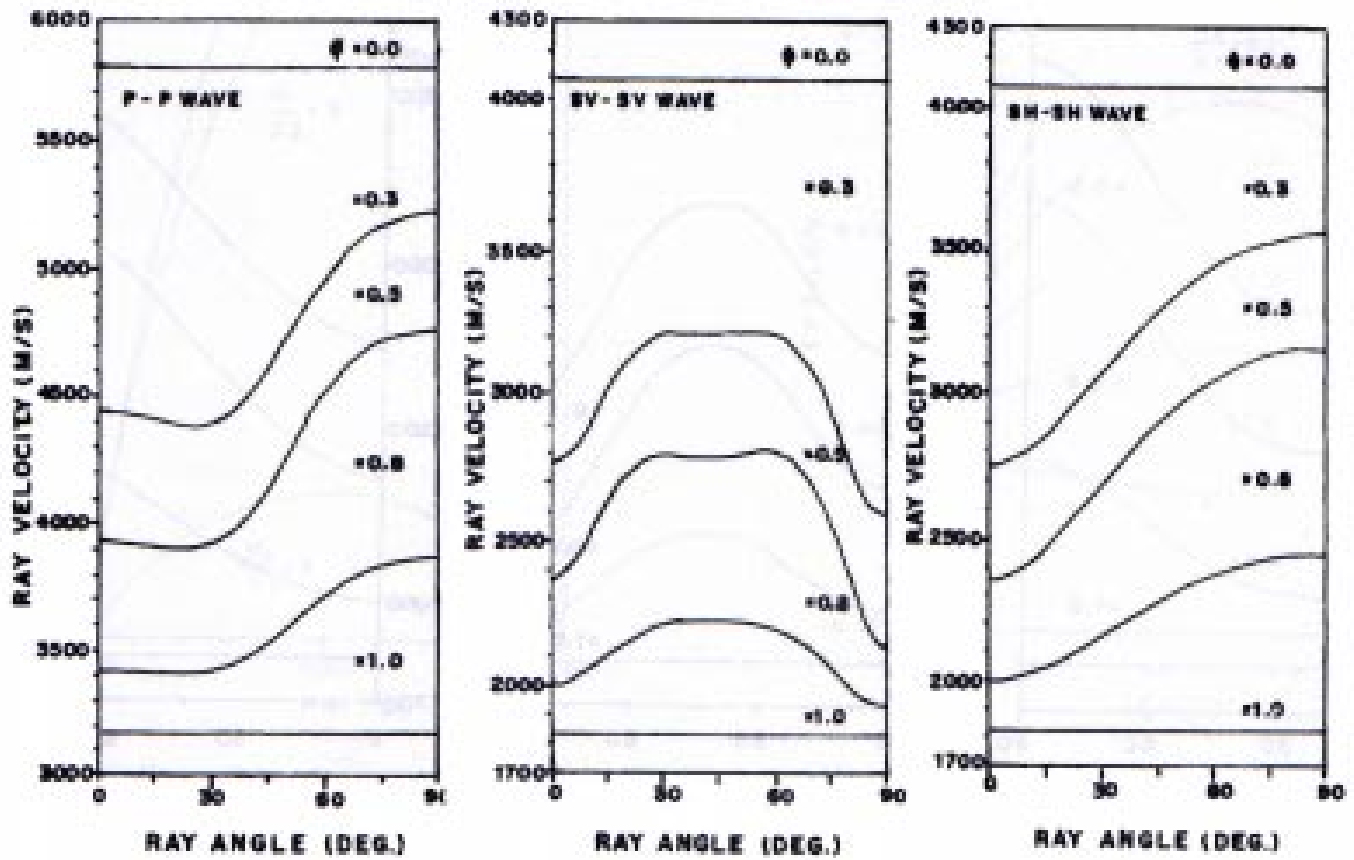

Fig 7. Variation of ray velocity with ray angle for different volume fraction of material 1 in the layeed stack 

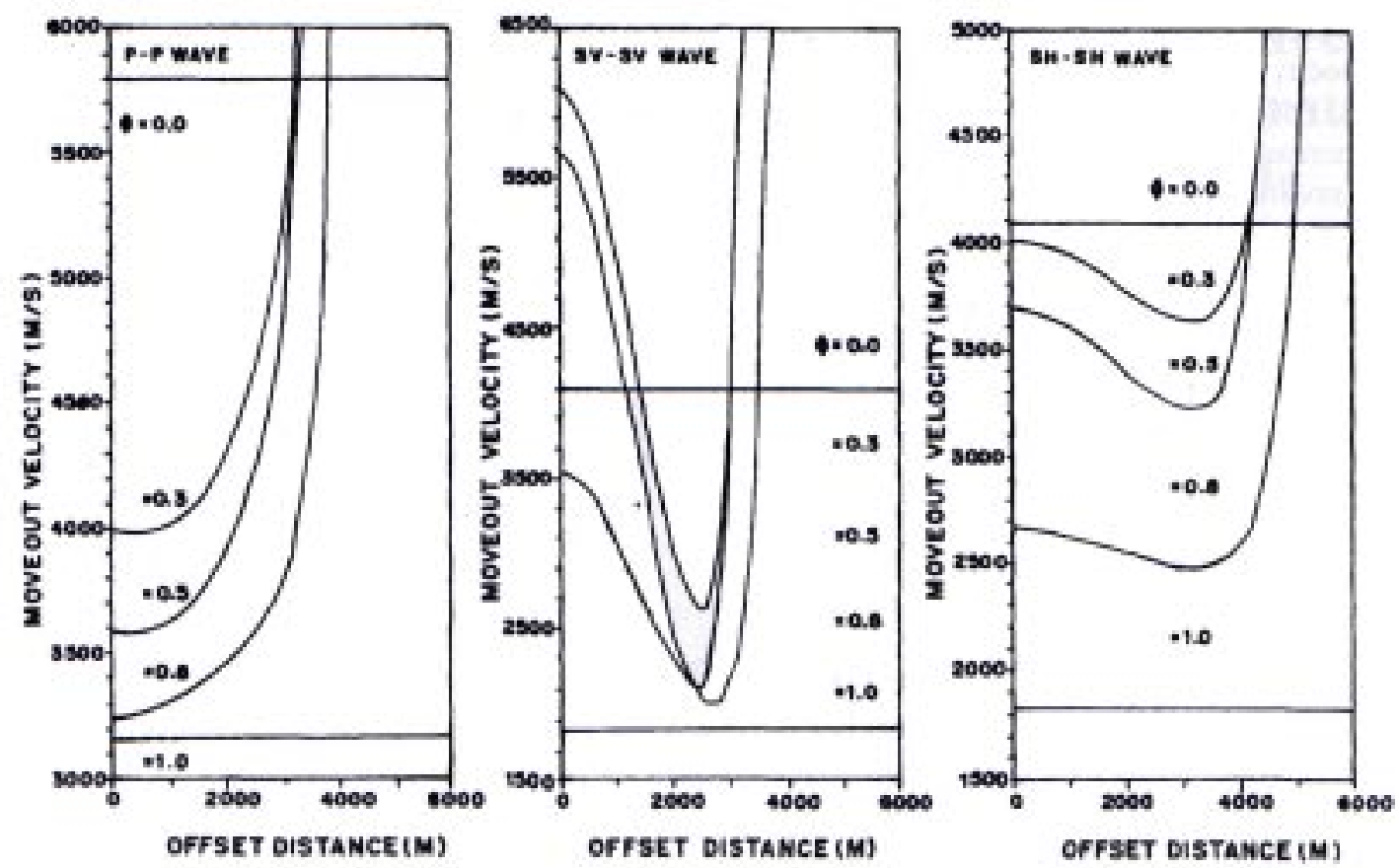

Fig 8. Variation of moveout velocity with offset distance for different volume fraction of material 1 in the layered stack

spread length $(0<\mathrm{X}<2 \mathrm{Z})$ in weakly anisotropic medium, phase angle and group angle have been equated as already discussed by Brown et al.,( 1991). The variation of ray velocity with ray angle has been computed using eqn.(11) and results are shown in Fig.(7). It is found that ray velocity follow similar trend as phase velocity with phase angle. The magnitude of ray velocity is found different from magnitude of phase velocity due to significant contribution of first order deriva- tive of phase velocity for quasi-P, quasi-S and transverse waves.

The analytical expression of moveout velocity given in eqn.(17) has been used to compute the variation of moveout velocity with offset distance at reflector depth of $3.0 \mathrm{~km}$ for different composition of two isotropic materials which makes a transversely isotropic media for all the three waves and results are shown in
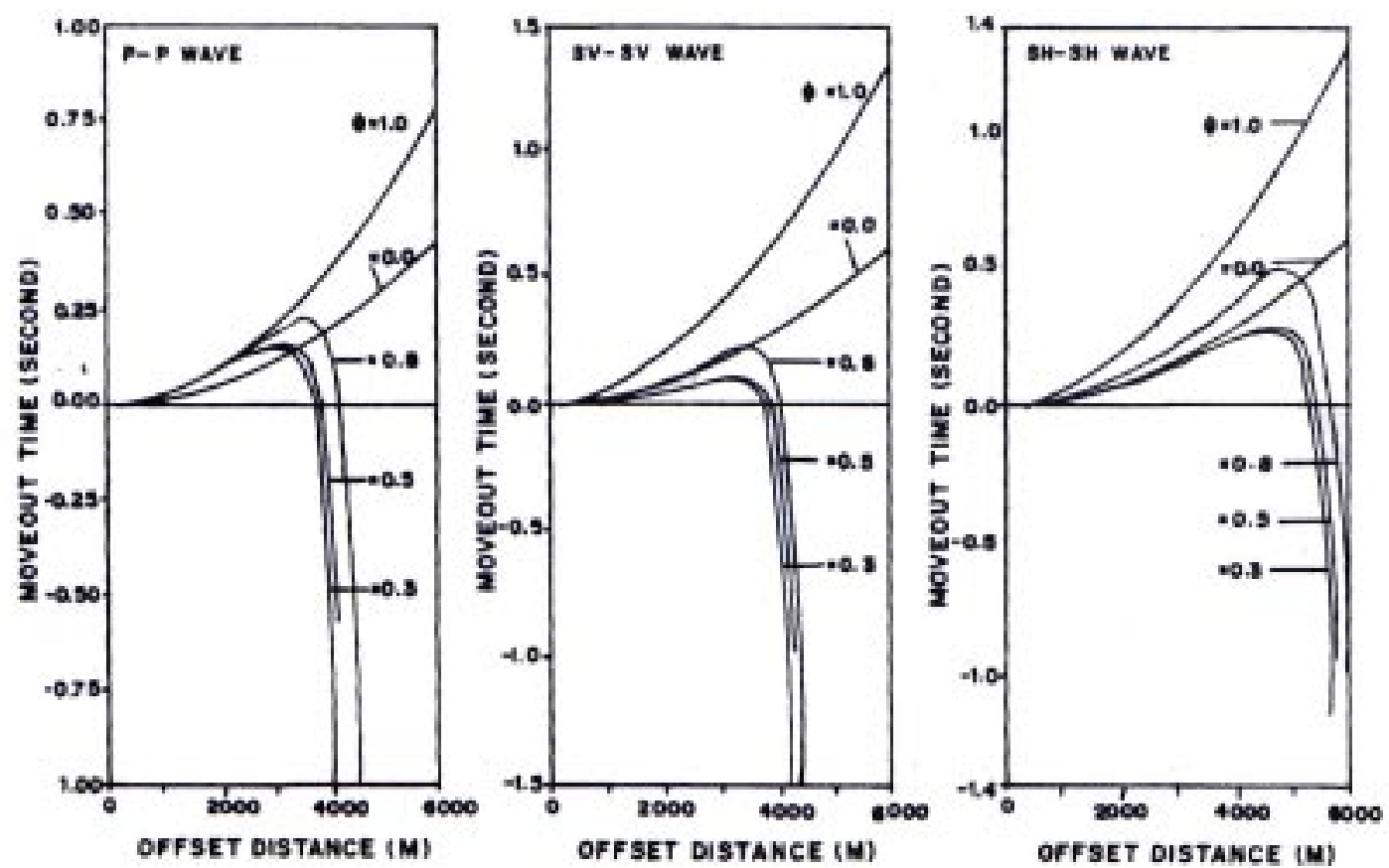

Fig 9. Variation of moveout travel time $\left(D t=t_{1}-t_{0}\right)$ with offset distance for different volume fraction of material 1 in the layered stack 
Fig.(8). At $f=0$ and 1 the moveout velocity is independent of offset distance and as $f$ increases the moveout velocity decreases for quasi-P, quasi$S$ and transverse waves. For $0<f<1$ moveout velocity increases slowly as offset increases and after a critical value of offset it increases exponentially. In case of quasi-S waves the NMO velocity for $f=0.3$ and 0.5 is even greater than the NMO velocity for $f=0$ and 1 . As offset increases moveout velocity decreases sharply for $0<f<1$ lies in between the NMO velocity for $f=0$ and 1 . With the further increase of offset distance moveout velocity decreases and after a certain value it follows similar trend as in case of other two waves.

Using the generalized analytical expression of travel time given in eqn.(21), the variation of moveout travel time $\left(D t=t_{1}-t_{\text {io }}\right)$ with offset distance have been computed for different composition of isotropic materials and results are shown in Fig.(9) for quasi-P, quasi-S and transverse waves respectively. It is found that the moveout travel time for quasi-P waves is minimum at $\mathrm{f}=0$ and maximum at $\mathrm{f}=1$ for $\mathrm{X}$ $<3000 \mathrm{~m}$. As volume fraction of material 1 ( $)$ increases moveout travel time decreases. For $f$ $=0$ and 1 the moveout travel time follows hyperbolic nature for all the three waves namely quasi-P, quasi-S and transverse waves at all offset distances whereas it becomes non-hyperbolic after certain offset distance for other composition of isotropic materials lying in the range $0<$ $\mathrm{f}<1$. In case of quasi-S waves the moveout travel time is always less than for $0<\mathrm{f}<1$ to the moveout travel time at $f=0$. This shows that the quasi-S waves are much more sensitive for the presence of anisotropy even at smaller offset also as compared to quasi-P and transverse waves. The moveout travel time of transverse waves also follows hyperbolic nature for lower offset distances at different $f$ lying between 0 and 1 . For higher offset distances the moveout travel time becomes non-hyperbolic in the presence ofanisotropy i.e., for $0<f<1$. Thus, it is seen that the moveout travel time changes significantly for finite offsets as volume fraction of isotropic materials changes for all the three waves namely quasi-P, quasi-S and transverse waves.

\section{Conclusions:}

The equivalent medium composed of two isotropic materials produces a highly anisotropic medium if two layers are having significant difference in shear moduli and ( $\mathrm{Vs} / \mathrm{Vp})$ ratio. This study has shown that the characteristics of seismic wave parameters such as ray velocity, moveout velocity and moveout travel time change significantly if wave is passing through a transversely isotropic medium which is composed of two homogeneous and isotropic materials. The interpretation of seismic waves passing through such medium may consequently lead to an inaccurate representation of earth's saurface if the presence of anisotropic is not taken into account properly. The analytical formulation given here may also be very useful when combined with migration and inversion algorithms where intensive computations are' required. This study also emphasizes that the knowledge of depositional systems ( geological formation) in association with the seismic interpretation of travel time in the presence ofanisotropy maybe more helpful in identifying hydrocarbon prospects in terms of source, spatial variability of reservoir bodies and entrapments.

\section{Acknowledgements:}

Authors are grateful to Oil and Natural Gas Corporation limited, India for providing the necessary information and infrastructural facilities to carryout this work. Authors are grateful to Director (Exploration), ONGC for according approval to publish this paper. Authors are grateful to Mr.A.G.Pramanik, Group General Manager (Exploration), Dr.C.H.Mehta, General Manager( Prog.), Mr.Gautam Sen, General Manager (Geophys.) and Mr.D.P. Sinha, Chief Geophysicist for their valuable guidance and useful comments.

\section{References:}

Backns, G:E.. 1962, Ling wave elastic ailiscitrcpv produced by horizontal layering, J.Geophys. Res.,V-67,4427-4440.

Beiryman, J.G., Crrechka,V and Berge, EA., 1997, Analysis of Thomsen parameters for finely layered VTI media, 67th SEG annual meeting 
and international exposition held at Dallas, USA, Expanded abstract, 941-944.

Brittan, J, Warner, M. andPratt, G., 1995, Anisotropic parameters of layered media $\mathrm{m}$ terms of composite elastic properties, Geophys., V-60, 1243-1248.

Brown, R.J., Lawton, D.C. and Cheadle. S.P., 1991, Scaled physical modeling of anisotropic wave propagation: Multi-offset profile over an ortho-rhombic medium, Geophys. J. international, 107, 693-702.

Gassmann, F., 1951, Uberdie elastizitatpiiroser medien, Veirteljabrassdirift der Naturtorsdiaidea Ges'ellscfaaft in Zurich, 96, 1-23.

Helbig, K.,1994, Foundation ofanisotropy for exploration seismics: Hand book of geophysical exploration, edited by Klans Helbig and S. Treitel, V-22, Pergamon pres.s.

Rommel, B.E., 1993, Approximate stacking velorities in weakly transversely isotropic layers, Canadian J. Exploration Geophys., V-29, 98105.
Singh. V, and Sanjay Klirnar, 1995, Origin of anisotropy and its effect on seismic exploraticn. Proceedings of GJS on exploration geophysics $m$ India held at Calcutta during Nov. 14-16, up. pub. No. 49, 265-278

Singh, V, Sanjay Kumar, Khanna, R.K. and D.Dutta, 1997a, Anisotropy and its role on structural imaging. Proceedings of Petrotech-97.V-2. 341-352.

Singh, V, 1997b, Sei.anic Anisotropy : An effective tool for hydrocarbon exploration, Geohorizons, V-2, No.1, 12-13.

Thomsen, L., 1986, Weak elastic anisotropy, Geophys., V-51, 1954-1966.

Tsvankm, 1. and Thom.senJ ., 1994, Non-hyperbolic reflection moveout in anisotropic media, Geophys. V-59, 1290-1304.

Van der Stoep, D.,1966, Velocity anisotropy measurements in wells, Geophys., V-31,900916. 